\title{
Theoretical Study on the Flow Generated by the Strike-Slip Faulting
}

\author{
Chi-Min Liu,, ${ }^{1,2}$ Ray-Yeng Yang, ${ }^{2,3}$ and Hwung-Hweng Hwung, \\ ${ }^{1}$ Division of Mathematics, General Education Center, Chienkuo Technology University, Changhua City 500, Taiwan \\ ${ }^{2}$ International Wave Dynamics Research Center, National Cheng Kung University, Tainan 701, Taiwan \\ ${ }^{3}$ Tainan Hydraulic Laboratory and Research Center of Ocean Environment and Technology, National Cheng Kung University, \\ Tainan 701, Taiwan \\ ${ }^{4}$ Department of Hydraulic and Ocean Engineering, National Cheng Kung University, Tainan 701, Taiwan
}

Correspondence should be addressed to Chi-Min Liu; cmliu@ctu.edu.tw

Received 25 April 2013; Accepted 22 May 2013

Academic Editors: G. Casula, E. Liu, and K. Maamaatuaiahutapu

Copyright (C) 2013 Chi-Min Liu et al. This is an open access article distributed under the Creative Commons Attribution License, which permits unrestricted use, distribution, and reproduction in any medium, provided the original work is properly cited.

The flow driven by the strike-slip faulting is theoretically analyzed in this paper. The surface of the strike-slip fault is generally near vertical, and the corresponding plates move in horizontal directions during the faulting. The focus of present paper is on the flow at the early stage when the faulting is activated. Standard procedures for deriving the exact solution of the induced flow are first demonstrated. Based on the derived solution, flows generated by three kinds of faulting are examined to observe and compare the evolution of velocity profiles and the corresponding kinetic energy. The results show that the flow energy rapidly decays as the speed of the moving plates begins to slow down. Moreover, mathematical methods proposed in this study provide a useful basis for related studies on not only geophysics, but also fluid mechanics, industry manufacturing, heat-conduction problems, and other possible applications.

\section{Introduction}

It is well known that many earthquakes are attributed to the fault motion. In general, there are two kinds of faults, the dip-strike and the slip-strike faults, which indicate that the relative movements on the fault plane are approximately vertical and horizontal, respectively. For oceanographers and ocean engineers, a great deal of attention is focused on not only the faulting properties but also the induced flow above the plates. From the viewpoint of energy, the dip-strike and slip-strike faults, respectively, result in the transfer of potential energy and kinetic energy from the moving plates to the fluid. As the dip-strike faulting usually transfers a considerable amount of potential energy into the above fluid, a tsunami wave is simultaneously generated on the ocean surface. Due to the catastrophic damage by the tsunami wave, related studies either on the generation or the propagation of tsunamis are quite abundant (see Ben-Menahem and Rosenman [1], Hammack [2], Okada [3], Dutykh and Dias $[4,5]$, and Saito and Furumura [6] for details). However, for the flow induced by the slip-strike faulting, it seems to receive much less attention from scientists.

For the sake of modeling the flow driven by the strikeslip faulting, Zeng and Weinbaum [7] may be the first who theoretically studied the flow induced by relatively moving half plates. The two half plates are assumed to move either in a constant speed or a harmonic oscillation. The steady-state solution to flow velocity is then obtained by employing some mathematical techniques. However, the steady-state solution cannot exactly capture the flow at the early stage because the transient component of the flow is totally ignored in their analysis. As the duration of the strike-slip faulting is usually short, Liu [8] theoretically solved the same problems by applying different mathematical methods and then acquired the exact solutions which can successfully describe the flow not only at larger times but also at smaller times. Later, Liu [9] further studied similar problems, while either the mass influx or outflux is allowed on the moving plates.

Though aforementioned papers have preliminary studied the topic considered in the present paper, some important 


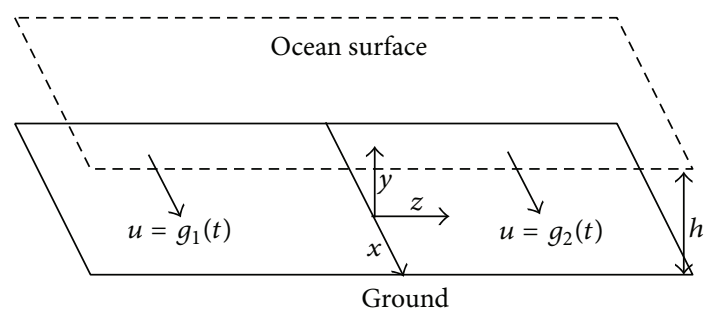

(a)

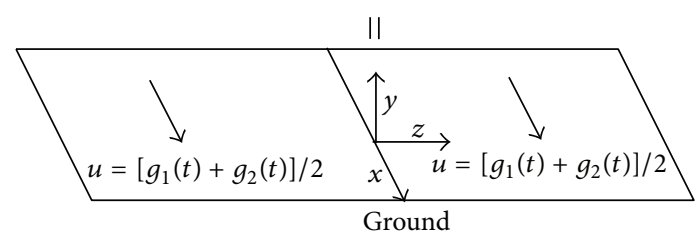

(b)

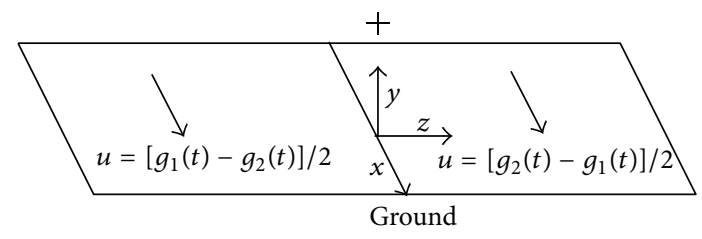

(c)

FIGURE 1: Definition sketches for (a) the original problem, (b) the first subproblem of one-dimensional flow, and (c) the second subproblem of two-dimensional flow.

aspects, however, have not yet been well investigated. First, standard procedures for solving the flow driven by arbitrary motion of plates have not been established even though the cases of the so-called extended Stokes' problems were investigated by Liu [8]. Besides, the kinetic energy which plays a more important role in assessing the influence of the flow on neighboring environment has not been calculated in previous studies. To this end, the primary goal of this study is to study these aspects. This paper is organized as follows. In Section 2, mathematical techniques and standard procedures are developed to derive the exact solution of the flow generated arbitrarily moving half plates. Based on the proposed methods, flows induced by three faulting types are, respectively, solved and the corresponding exact solutions are given in Section 3. The velocity evolution and kinetic energy are also analyzed. Finally, important results and future works are briefly concluded in Section 4.

\section{Theoretical Analysis}

For a viscous flow driven by the moving plates, the difficulty of solving the exact solution greatly depends on how complex the plates shift is. The most well-known case is the Stokes' problems $[10,11]$ describing a flow generated by a single plate moving in either a constant speed or a harmonic oscillation. The Stokes' problem depicts a one-dimensional flow. One only needs to apply the Laplace transform to acquire the exact solution which provides an accurate prediction of flow at any time. The problem considered is depicted in Figure 1(a).
The fault surface is assumed to be located at the $x-y$ plane and extends from $x=-\infty$ to $x=\infty$ and from $y=$ 0 to $y=-\infty$. A viscous fluid of depth $h$ above the $x-z$ plane remains motionless for $t<0$. When $t>0$, the flow is suddenly driven by two plates moving in the opposite directions along the $x$ coordinate. The speeds of the negative$z$ and positive- $z$ plates are represented by arbitrarily timedependent functions, $g_{1}(t)$ and $g_{2}(t)$. Since the induced flow is of two spatial dependences $y$ and $z$, more mathematical techniques besides the integral transform used in solving Stokes' problems are required to derive the solution. The most important technique is to suitably decompose the original problem into two subproblems, as shown in Figures 1(b) and 1(c). For the first subproblem, the induced flow is onedimensional since the motion of two plates is uniform and equal to $\left[g_{1}(t)+g_{2}(t)\right] / 2$. Based on the solutions of the classical Stokes' first and second problems, the exact solution is readily acquired by using the Fourier analysis. Therefore, the following analysis will focus on analyzing the second subproblem. In this problem that two plates move in the same speed, $\left[g_{1}(t)-g_{2}(t)\right] / 2$, but in the opposite direction implying the induced flow will be antisymmetrical with respect to the $x-y$ plane. Therefore, only the analysis of the flow in the positive- $z$ domain is required to understand the whole flow. The momentum equation along the $x$ direction, boundary conditions and initial condition in the positive- $z$ domain are shown as follows:

$$
\begin{gathered}
u_{t}=v\left(u_{y y}+u_{z z}\right), \\
u(y=0)=g(t), \\
u_{y}(y=h)=0, \\
u(z=0)=0, \\
u(t=0)=0,
\end{gathered}
$$

where $v$ is the kinematic viscosity, the subscript denotes the differentiation, and

$$
g(t) \equiv \frac{1}{2}\left[g_{2}(t)-g_{1}(t)\right]
$$

denotes the plate speed. It is noted that (4) results from that the induced flow is antisymmetrical with respect to the $x-z$ plane. In addition, for the sake of employing the integral transform with suitable boundary conditions, (3) is replaced by

$$
u(y=2 h)=g(t)
$$

To obtain the exact solution, several mathematical techniques are required and will be briefly demonstrated after. Firstly, the Laplace transform,

$$
\widehat{u}(y, z, s)=\int_{0}^{\infty} u(y, z, t) \cdot e^{-s t} d s,
$$

is applied to the previous PDE system with the help of the initial condition, (5). Next, one has to shift the transformed 
variable $\widehat{u}$ to a new variable $u^{*}$ where $u^{*}=\widehat{u}-\widehat{g}(s)$. The purpose of this shifting is to make the resulting boundary conditions $u^{*}(y=0)$ and $u^{*}(y=2 h)$ be zero. With the help of the shifted boundary conditions, the Fourier sine transform defined as

$$
\tilde{u}(s, n, z)=\int_{0}^{2 h} u^{*}(s, y, z) \sin \left(\frac{n \pi}{2 h} y\right) d y
$$

is subsequently performed to the PDE system. The final result is

$$
\widetilde{u}_{z z}-\left(\frac{s}{v}+\frac{n^{2} \pi^{2}}{4 h^{2}}\right) \widetilde{u}=\frac{2 h}{n \pi \nu}\left(1-(-1)^{n}\right) s \widehat{g}(s),
$$

with boundary conditions

$$
\begin{gathered}
\tilde{u}(z=0)=-\frac{2 h}{n \pi}\left(1-(-1)^{n}\right) \cdot \widehat{g}(s), \\
\tilde{u}(z=\infty)=\text { finite value. }
\end{gathered}
$$

Now the solution to $\tilde{u}$ can be determined by solving (10) to (12). It reads

$$
\begin{aligned}
\tilde{u}= & \frac{2 h\left(1-(-1)^{n}\right)}{n \pi \nu\left(4 h^{2} s v^{-1}+n^{2} \pi^{2}\right)} \\
\times & {\left[\left(4 h^{2}-4 h^{2} s v^{-1}-n^{2} \pi^{2}\right)\right.} \\
& \left.\quad \times \exp \left(-\sqrt{\frac{s}{v}+\frac{n^{2} \pi^{2}}{4 h^{2}}} \cdot z\right)-4 h^{2}\right] \cdot s \hat{g}(s) .
\end{aligned}
$$

After taking the inverse spatial and time transforms, the exact solution of the flow velocity $u$ in the positive- $z$ domain can be obtained. As for the negative- $z$ domain, the solution is given by the relation $u(-z)=-u(z)$ due to the antisymmetrical concept.

\section{Flow Development and Kinetic Energy}

From the previous section, the flow velocity can be exactly calculated at any spatial position and at any time. In this section, the evolution of flow velocity and the corresponding kinetic energy will be explored to understand the fundamental characteristics of the induced flows. For the sake of simplification, only the second subsystem displayed in Figure 1(c) is investigated herein. For simulating the possible strike-slip faulting and the induced flow, three faulting types are considered:

(A) uniform motion:

$$
g(t)=u_{0}, \quad \text { at } 0 \leq t \leq t_{0}
$$

(B) linear decay:

$$
g(t)=u_{0}\left(1-\frac{t}{t_{0}}\right), \quad \text { at } 0 \leq t \leq t_{0},
$$

(C) exponential decay:

$$
g(t)=u_{0} \exp (-\alpha t), \quad \text { at } 0 \leq t \leq t_{0},
$$

where $u_{0}$ is the constant velocity and $t_{0}$ the duration of the faulting. The exact solutions of faulting types (A), (B) and (C) are respectively shown

$$
\begin{aligned}
& U=1+\sum_{n=1}^{\infty} 2 \cdot \frac{1-(-1)^{n}}{n \pi} \sin \left(\frac{n \pi Y}{2}\right) \\
& \left\{-\exp \left(-\frac{n^{2} \pi^{2} T}{4}\right) \operatorname{erf}\left(\frac{Z}{2 \sqrt{T}}\right)\right. \\
& \left.-\int_{0}^{T} \frac{Z}{2 \sqrt{\pi\left(T^{\prime}\right)^{3}}} \exp \left(-\frac{n^{2} \pi^{2} T^{\prime}}{4}-\frac{Z^{2}}{4 T^{\prime}}\right) d T^{\prime}\right\},
\end{aligned}
$$

$$
\begin{aligned}
U=1 & -\frac{T}{T_{0}}+\sum_{n=1}^{\infty} 2 \cdot \frac{1-(-1)^{n}}{n \pi} \sin \left(\frac{n \pi Y}{2}\right) \\
& \cdot\left\{\frac{4}{n^{2} \pi^{2} T_{0}}-\left(1+\frac{4}{n^{2} \pi^{2} T_{0}}\right) \exp \left(-\frac{n^{2} \pi^{2} T}{4}\right) \operatorname{erf}\left(\frac{Z}{2 \sqrt{T}}\right)\right. \\
& +\int_{0}^{T}\left(\frac{T-T^{\prime}}{T_{0}}-1-\frac{4}{n^{2} \pi^{2} T_{0}}\right) \frac{Z}{2 \sqrt{\pi\left(T^{\prime}\right)^{3}}} \\
& \left.\times \exp \left(-\frac{n^{2} \pi^{2} T^{\prime}}{4}-\frac{Z^{2}}{4 T^{\prime}}\right) d T^{\prime}\right\}
\end{aligned}
$$

$$
\begin{aligned}
U= & \exp (-A T)+\sum_{n=1}^{\infty} 2 \cdot \frac{1-(-1)^{n}}{n \pi} \sin \left(\frac{n \pi Y}{2}\right) \\
& \cdot\left\{\frac{-A}{A-\left(n^{2} \pi^{2} / 4\right)} \exp (-A T)+\frac{n^{2} \pi^{2} / 4}{A-\left(n^{2} \pi^{2} / 4\right)}\right. \\
& \times \exp \left(-\frac{n^{2} \pi^{2}}{4} T\right) \operatorname{erf}\left(\frac{Z}{2 \sqrt{T}}\right) \\
& +\frac{n^{2} \pi^{2} / 4}{A-\left(n^{2} \pi^{2} / 4\right)} \int_{0}^{T} \frac{Z}{2 \sqrt{\pi\left(T^{\prime}\right)^{3}}} \\
& \left.\times \exp \left[-A\left(T-T^{\prime}\right)-\frac{n^{2} \pi^{2} T^{\prime}}{4}-\frac{Z^{2}}{4 T^{\prime}}\right] d T^{\prime}\right\},
\end{aligned}
$$

where the dimensionless variables written in capitals are scaled as

$$
\begin{gathered}
U=\frac{u}{u_{0}}, \quad Y=\frac{y}{h}, \quad Z=\frac{z}{h}, \\
T=\frac{v t}{h^{2}}, \quad T_{0}=\frac{v t_{0}}{h^{2}}, \quad A=\frac{h^{2} \alpha}{v} .
\end{gathered}
$$




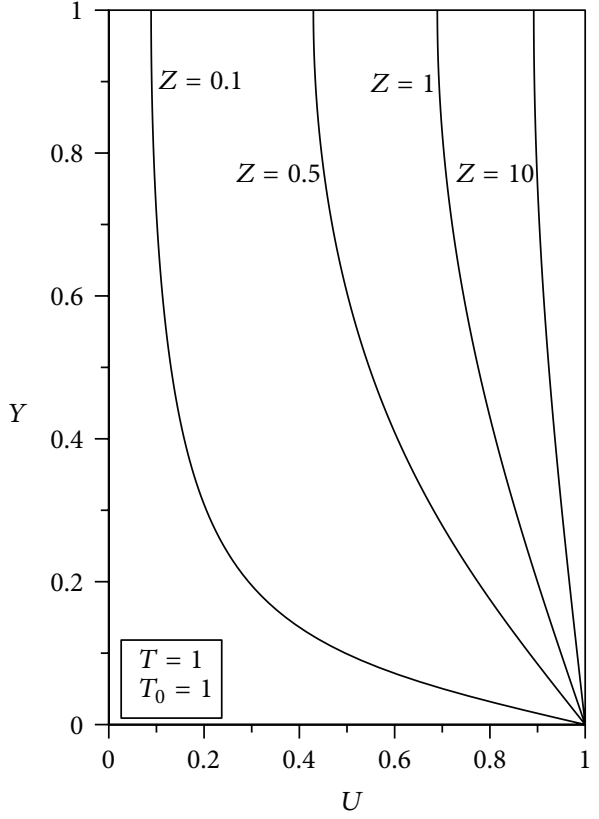

FIgURE 2: Velocity profiles at various $Z$ sections at $T=1$ for the faulting type A.

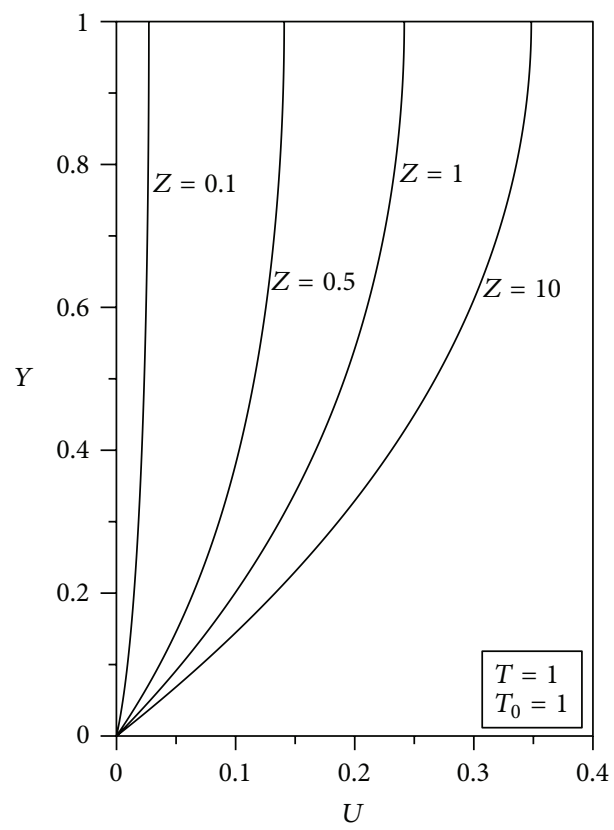

FIgURE 3: Velocity profiles at various $Z$ sections at $T=1$ for the faulting type $\mathrm{B}$.

Without loss of generality, the duration of faulting $T_{0}$ is assumed to be unity in the following analyses. The velocity profiles at various $Z$ sections at a fixed time $T=1$ (the end time of the faulting) are first calculated. Results of faulting types (A), (B), and (C) are, respectively, drawn in Figures 2,3 , and 4 . It is clearly seen that the velocity profile at smaller $Z$ section develops more slowly than that at larger $Z$ section due to the influence of the reverse flow in the

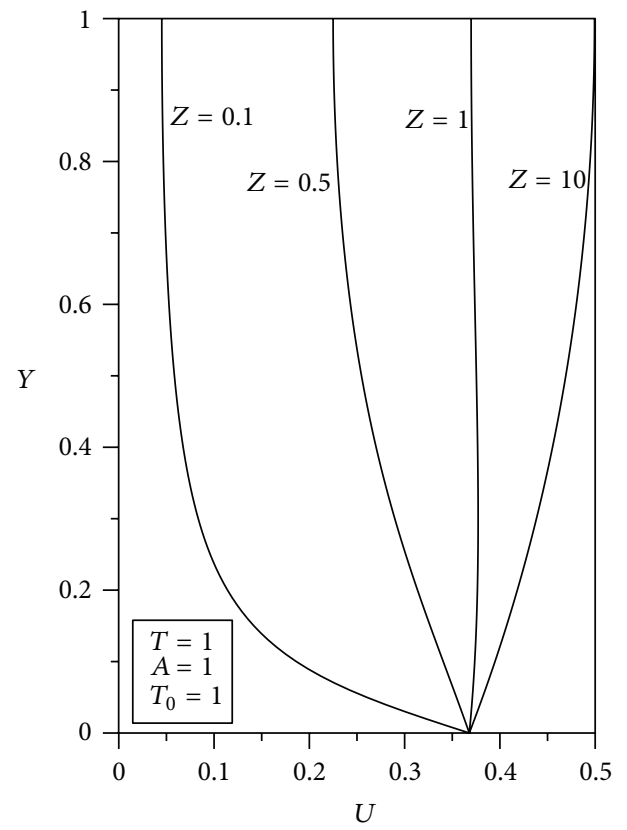

FIgURE 4: Velocity profiles at various $Z$ sections at $T=1$ for the faulting type $\mathrm{C}$.

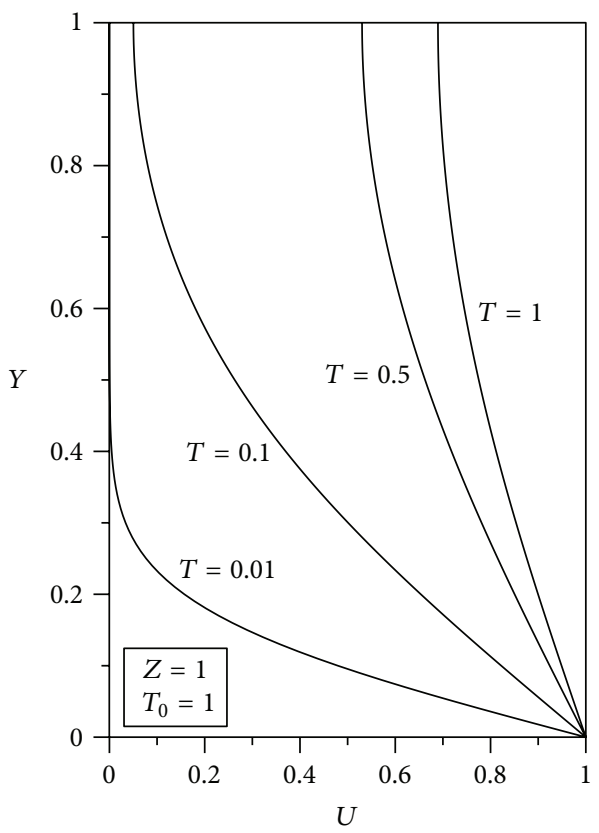

FIgURE 5: Velocity evolution at $Z=1$ for the faulting type A.

negative- $Z$ domain. This demonstrates a truth that the fluid near the fault moves more slowly than that far from the fault. Next, Figure 5 depicts the flow development during the faulting $(0 \leq T \leq 1)$ for the faulting type (A). At a fixed section $Z=1$, the velocity profile gradually grows and approaches the plate velocity since the energy is continuously transferred from the plate into the fluid. However, the flow developments of the faulting types (B) and (C) are quite different from that of faulting type (A), as shown in 


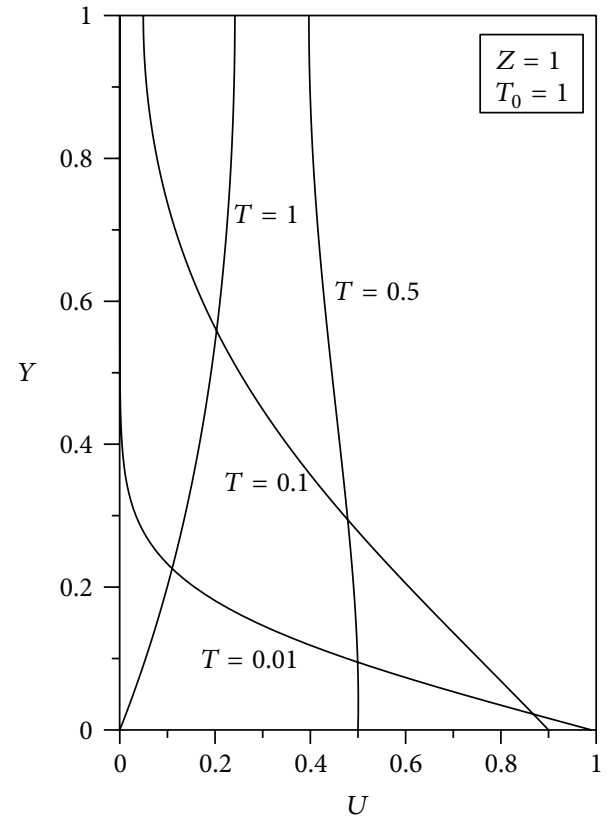

FIgURE 6: Velocity evolution at $Z=1$ for the faulting type B.

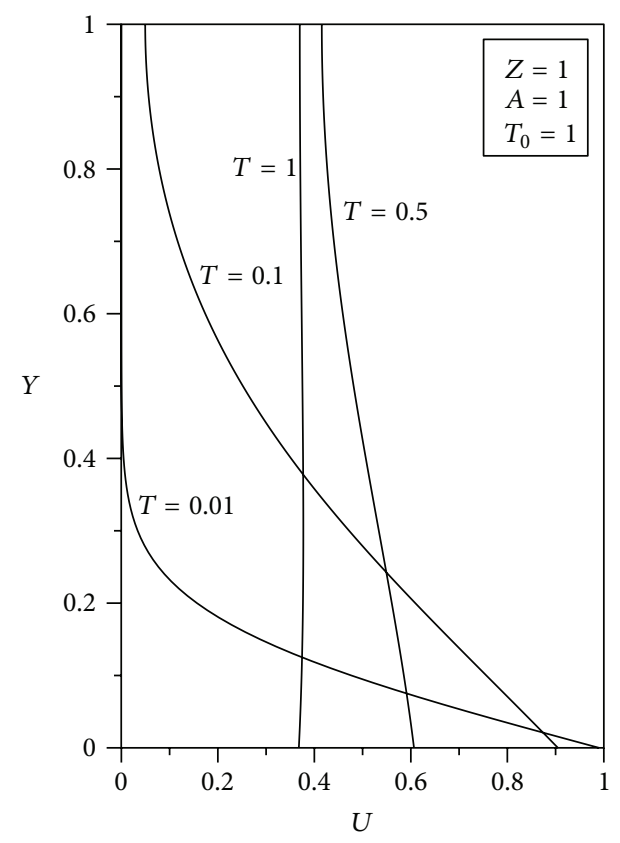

Figure 7: Velocity evolution at $Z=1$ for the faulting type C.

Figures 6 and 7. Since the plate speeds of faulting types (B) and $(\mathrm{C})$ decay linearly and exponentially, the velocity will rapidly grow at the early stage of the faulting and then quickly decay due to the effects of viscosity.

Next, the following integral

$$
E(Z, T) \equiv \frac{1}{2} \int_{0}^{1} U^{2} d y
$$

is defined to measure the kinetic energy per area. In comparison with velocity profiles, exploring the kinetic energy

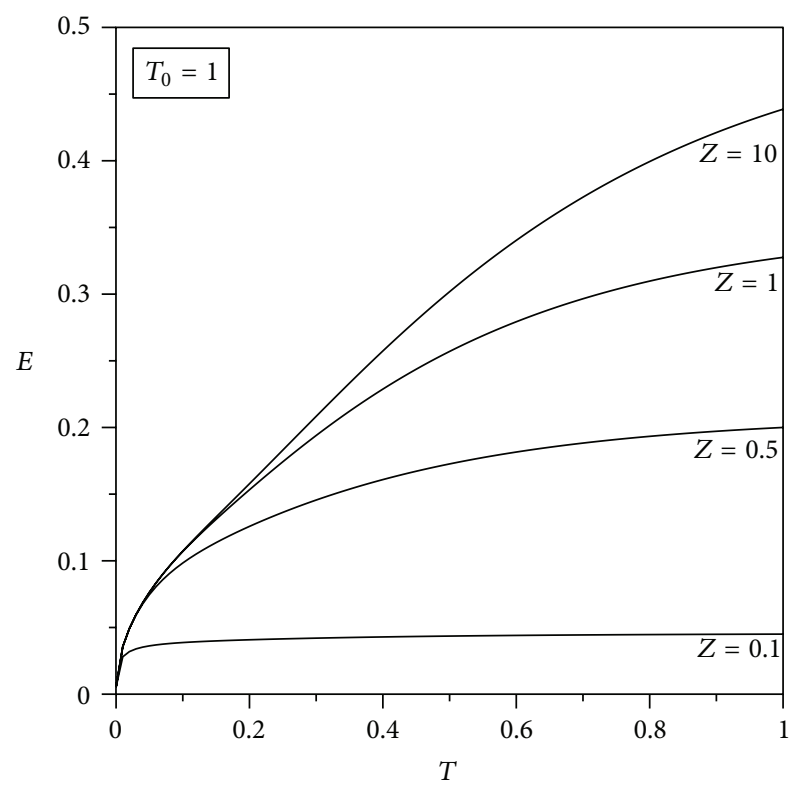

FIGURE 8: Relation between kinetic energy and time for the faulting type A.

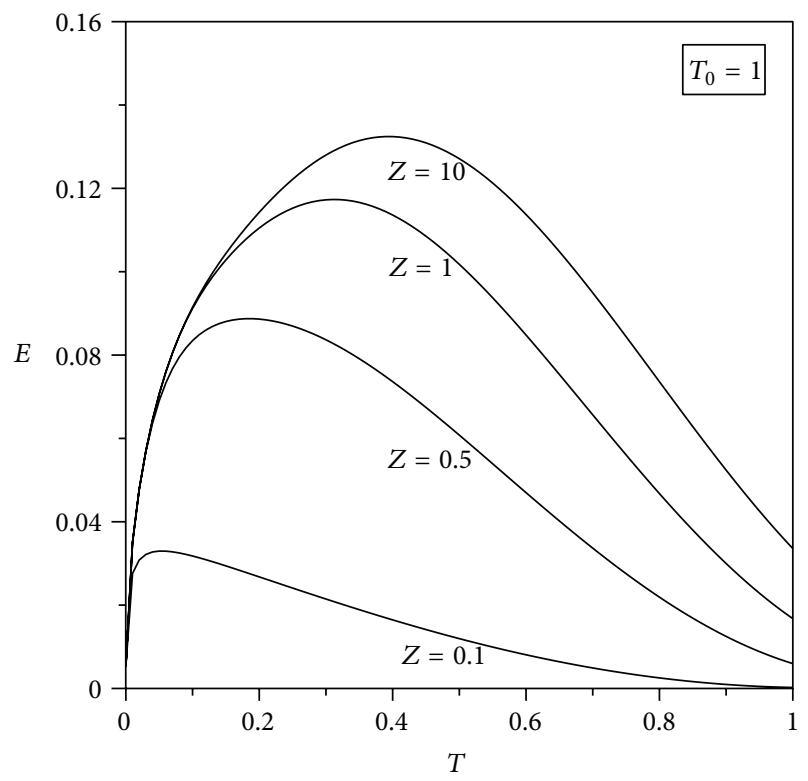

FIGURE 9: Relation between kinetic energy and time for the faulting type B.

can provide a much clearer understanding of the potential impact of the earthquake-induced flow on the neighboring region. Figure 8 shows the development of the kinetic energy of faulting type (A) at various $Z$ sections during the faulting. Similar to the velocity development, the kinetic energy at larger $Z$ section is greater than that at smaller $Z$ section. As the energy is continuously transferred into the fluid, the kinetic energy will gradually grow and approach a finite value before the faulting ends. As for results of faulting types (B) and $(C)$, the kinetic energy will reach a maximum value and then decay during the faulting, as shown in Figures 9 and 10. 


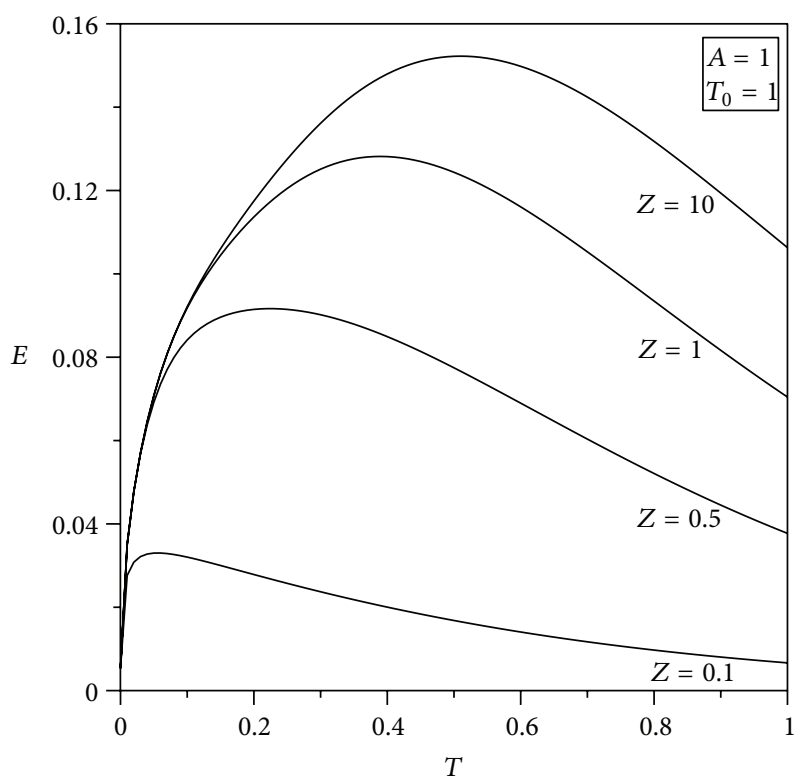

FIGURE 10: Relation between kinetic energy and time for the faulting type C.

Previous results indicate that the faster the faulting decays, the faster the flow energy attenuates.

\section{Conclusions}

The flow driven by the strike-slip faulting is theoretically analyzed in this paper. The focus is on the flow at the early stage when the faulting is activated. Standard mathematical procedures for deriving the exact solutions are provided. Three types of faulting are also investigated to calculate the velocity evolution and the corresponding kinetic energy. Results show that the kinetic energy rapidly decays as the speed of the moving plates begins to slow down. Based on present results, some suggestions and future works are briefly demonstrated later.

Though the flow after the faulting is not solved in this paper, it is not difficult to calculate it by using mathematical methods described later. The velocity profiles at $T=T_{0}$ (the end time of the faulting) have to be regarded as a new initial condition. The subsequent flow can be solved by applying integral transforms to the new PDE system. It seems quite straightforward and easy to perform the calculation. However, as the initial condition is no longer zero (e.g, substituting $T=T_{0}$ into (17) as new initial conditions), it generally results in the difficulty in performing the integral transform and its inversion. Due to the rapid decay of kinetic energy during the faulting, as shown in Figures 9 and 10 , it is reasonable to predict that the flow after the faulting will also decay rapidly since no external energy comes in. It indicates that the influence of the induced flow on neighboring structures and environment is much greater at the early stage of the faulting than that at longer times.

In addition to the faulting types considered in this paper, the flow induced by other types of faulting can be readily analyzed. Based on the present paper and that of Liu [8], the flow driven by arbitrary motion of two half plates can be theoretically calculated by using the Fourier analysis. Moreover, the procedures and mathematical techniques introduced herein can be applied to the case of non-Newtonian fluids. One needs to modify the constitutive equation (momentum equation) by including the rheological properties of fluids. As there exist a wide variety of non-Newtonian fluids, other mathematical techniques for dealing with additional rheological terms appearing in the constitutive equation may be necessary to derive the exact solution. The case of an Oldroyd-B fluid for similar problems was studied by Liu [12] who additionally employed the technique of the series expansion due to the unavailability of the inversed transforms.

Finally, mathematical methods used in this study can be applied to other fields. For example, the PDE system of the heat-conduction problem will be completely identical to (1) by modifying the velocity to temperature and the viscosity to conduction coefficient. In conclusion, the present study contributes to not only the theoretical solution of the earthquake-induced flow but also the related analyses in fluid mechanics, industry manufacturing, chemical engineering, and other fields.

\section{Acknowledgment}

The research funding provided by National Science Council of Taiwan with Contracts NSC 101-2221-E-270-001-MY2 and NSC 102-2911-I-006-302 is acknowledged.

\section{References}

[1] A. Ben-Menahem and M. Rosenman, "Amplitude patterns of tsunami waves from submarine earthquakes," Journal of Geophysical Research, vol. 77, no. 17, pp. 3097-3128, 1972.

[2] J. Hammack, "A note on tsunamis: their generation and propagation in an ocean of uniform depth," Journal of Fluid Mechanics, vol. 60, pp. 769-799, 1973.

[3] Y. Okada, "Surface deformation due to shear and tensile faults in a half-space," Bulletin of the Seismological Society of America, vol. 75, no. 4, pp. 1135-1154, 1985.

[4] D. Dutykh and F. Dias, "Tsunami generation by dynamic displacement of sea bed due to dip-slip faulting," Mathematics and Computers in Simulation, vol. 80, no. 4, pp. 837-848, 2009.

[5] D. Dutykh and F. Dias, "Energy of tsunami waves generated by bottom motion," Proceedings of the Royal Society A, vol. 465, no. 2103, pp. 725-744, 2009.

[6] T. Saito and T. Furumura, “Three-dimensional tsunami generation simulation due to sea-bottom deformation and its interpretation based on the linear theory," Geophysical Journal International, vol. 178, no. 2, pp. 877-888, 2009.

[7] Y. Zeng and S. Weinbaum, "Stokes problems for moving halfplanes," Journal of Fluid Mechanics, vol. 287, pp. 59-74, 1995.

[8] C.-M. Liu, "Complete solutions to extended Stokes' problems," Mathematical Problems in Engineering, vol. 2008, Article ID 754262, 18 pages, 2008.

[9] C.-M. Liu, "Extended stokes' problems for relatively moving porous half-planes," Mathematical Problems in Engineering, vol. 2009, Article ID 185965, 10 pages, 2009. 
[10] G. G. Stokes, "On the effect of the internal friction of fluids on the motion of pendulums," Transactions of the Cambridge Philosophical Society, vol. 9, pp. 8-106, 1851.

[11] C.-M. Liu and I.-C. Liu, "A note on the transient solution of Stokes' second problem with arbitrary initial phase," Journal of Mechanics, vol. 22, no. 4, pp. 349-354, 2006.

[12] C.-M. Liu, "Another approach to the extended Stokes' problems for the Oldroyd-B fluid," ISRN Applied Mathematics, vol. 2012, Article ID 274914, 14 pages, 2012. 

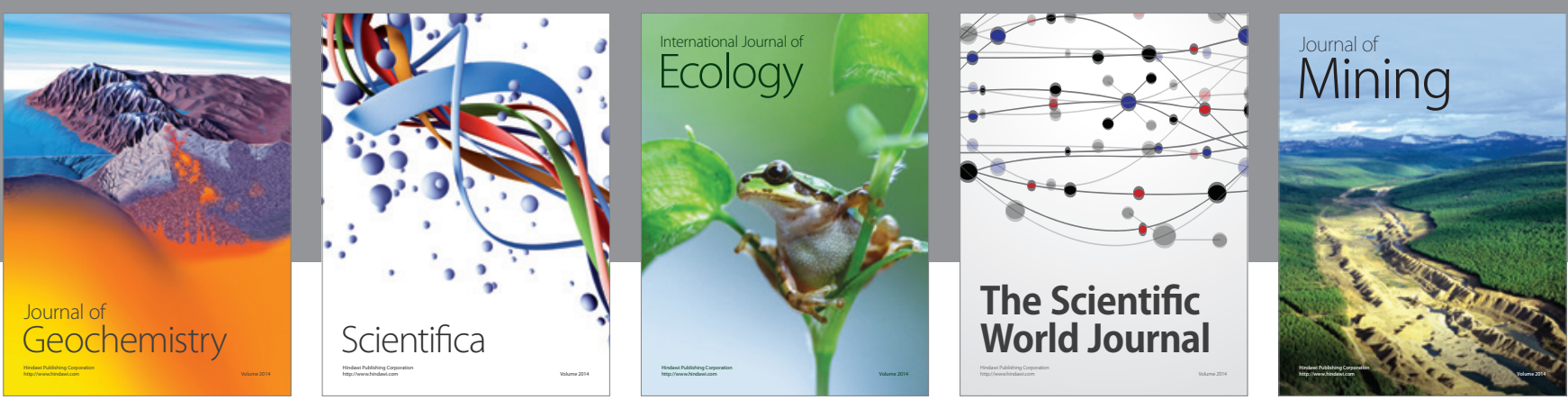

The Scientific World Journal
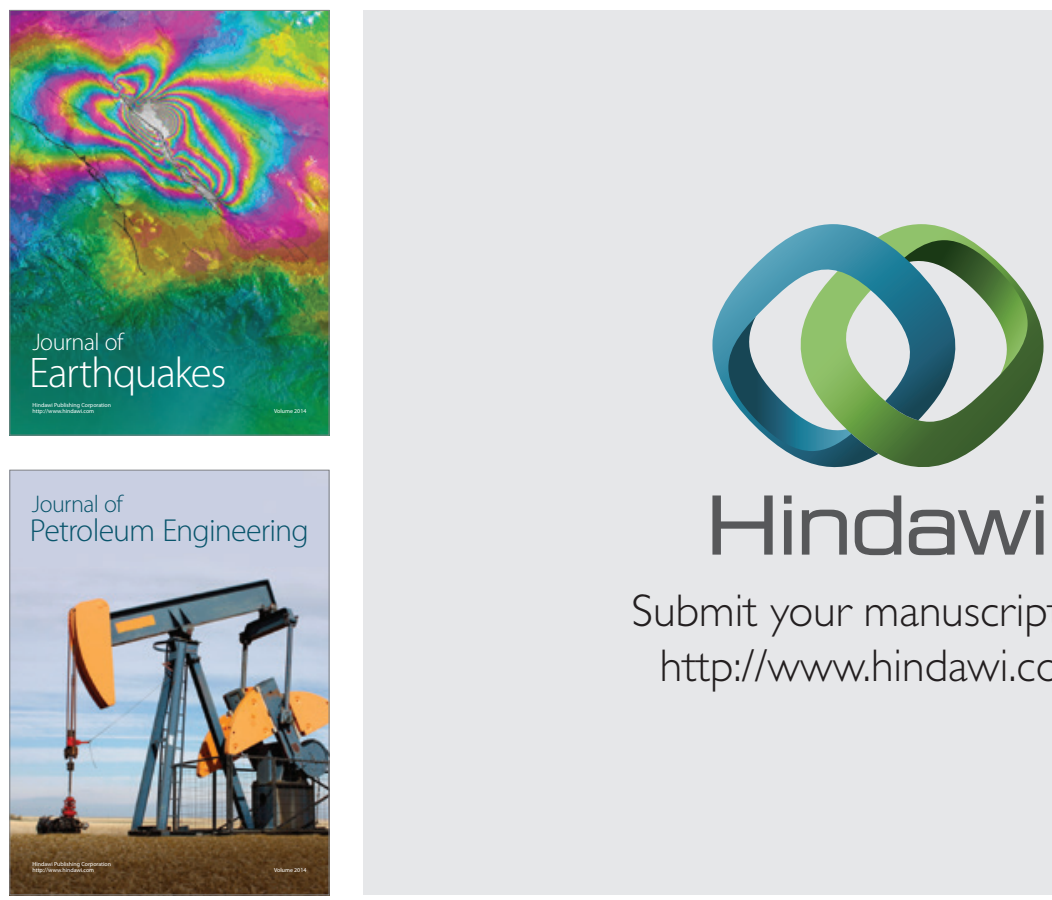

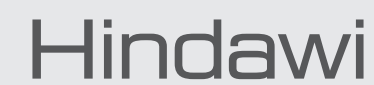

Submit your manuscripts at

http://www.hindawi.com
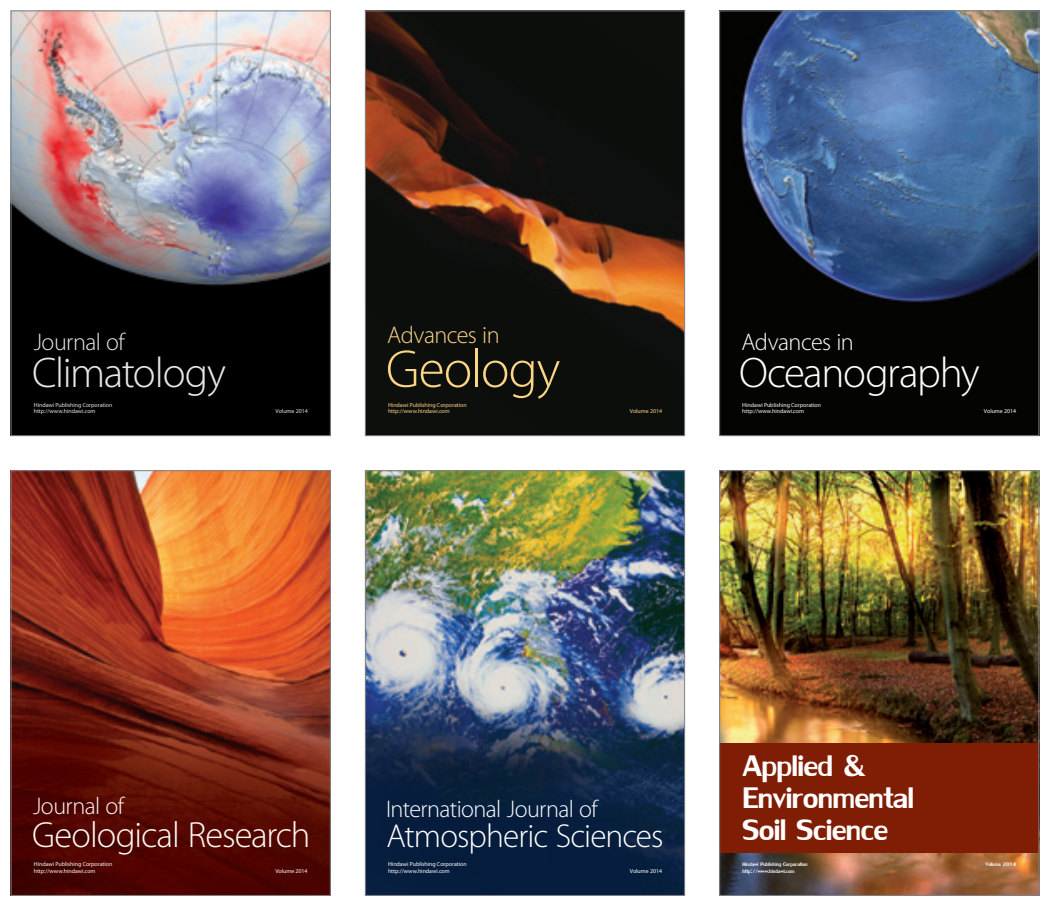
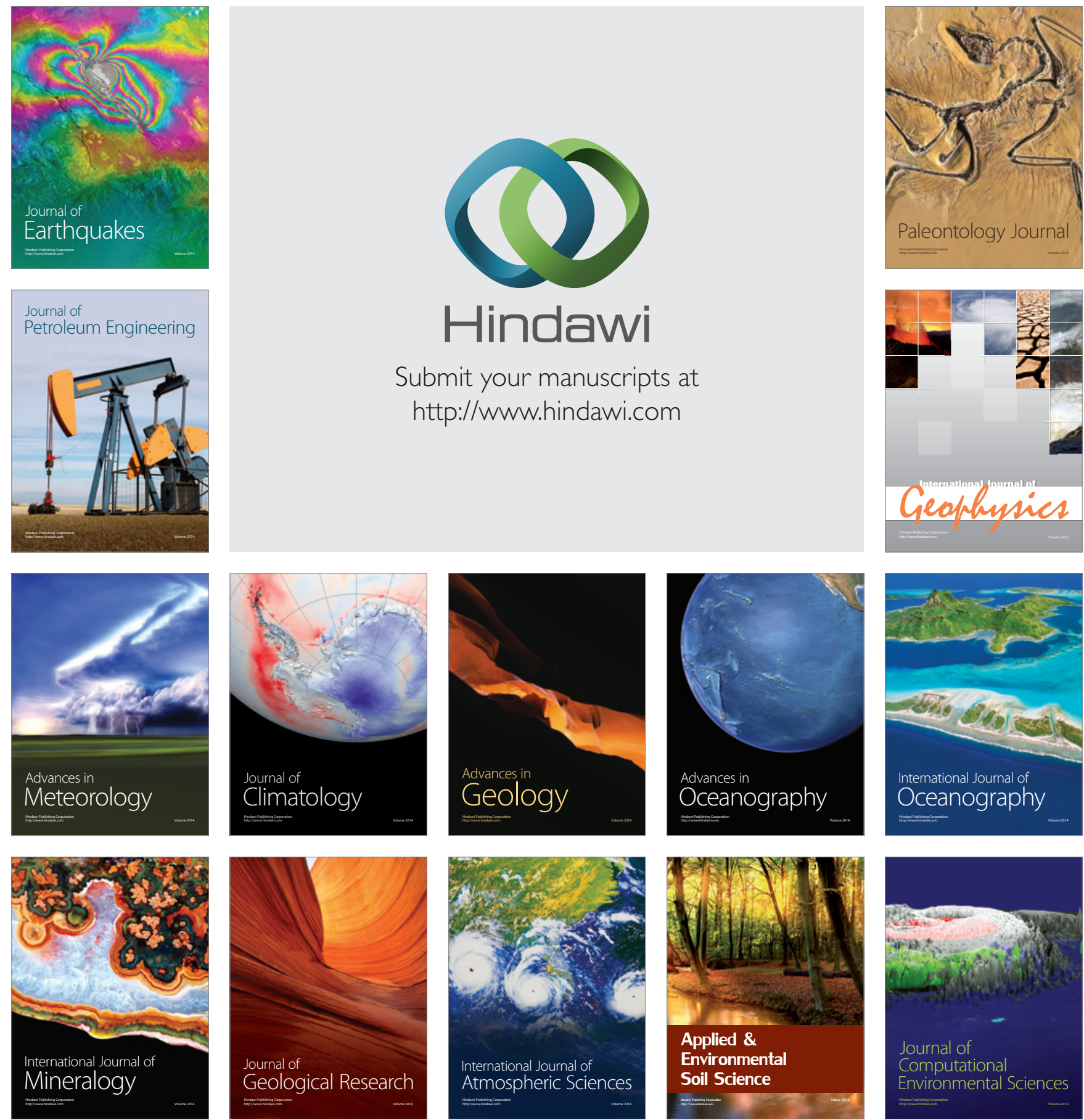